\title{
Differential Regulation and Correlation between Galectin-9 and anti-CCP antibody (ACPA) in Rheumatoid Arthritis Patients
}

\section{Yuya Fujita}

Fukushima Kenritsu Ika Daigaku

\section{Tomoyuki Asano}

Fukushima Kenritsu Ika Daigaku

Naoki Matsuoka

Fukushima Kenritsu Ika Daigaku

Jumpei Temmoku

Fukushima Kenritsu Ika Daigaku

Shuzo Sato

Fukushima Kenritsu Ika Daigaku

Haruki Matsumoto

Fukushima Kenritsu Ika Daigaku

Makiko Yashiro Furuya

Fukushima Kenritsu lka Daigaku

\section{Eiji Suzuki}

Fukushima Kenritsu Ika Daigaku

Hiroshi Watanabe

Fukushima Kenritsu Ika Daigaku

Atsushi Kawakami

Nagasaki Daigaku Byoin

Kiyoshi Migita ( $\nabla$ migita@fmu.ac.jp )

Fukushima Medical University School of Medicine

\section{Research article}

Keywords: Anti-citrullinated peptide antibody, galectin-9, rheumatoid arthritis, T cell Immunoglobulin Domain and Mucin-3

Posted Date: January 15th, 2020

DOI: https://doi.org/10.21203/rs.2.20926/v1 
License: (c) (i) This work is licensed under a Creative Commons Attribution 4.0 International License. Read Full License

Version of Record: A version of this preprint was published at Arthritis Research \& Therapy on April 15th, 2020. See the published version at https://doi.org/10.1186/s13075-020-02158-3. 


\section{Abstract}

Background: Galectin-9 (Gal-9) is involved in the regulatory process of immune responses or inflammation. The aim of the present study is to characterize circulating Gal-9 in patients with rheumatoid arthritis (RA) and its relationship with RA disease activity and phenotype.

Methods: A total 116 RA patients and age-matched healthy controls $(n=31)$ were included in this study. Disease activity of RA patients were determined by Disease Activity Score of 28 joint scoring system (DAS28-ESR). Levels of Galectin-9 in serum were determined by enzyme-linked immunosorbent assay (ELISA).

Results: Serum levels of Gal-9 were significantly higher in patients with RA compared to those in controls (median $7577 \mathrm{pg} / \mathrm{ml}$; [interquartile range (IQR); 5570-10201] versus $4738 \mathrm{~g} / \mathrm{ml}$ [IQR; 4267-5630], $p=0.001)$. Higher levels of serum Gal-9 were observed predominantly in RA patients with RA-ILD (9606 pg/ml [IQR: 8522-12167] versus 7078 pg/ml [IQR: 5225-9447], $p<0.001)$ or advanced joint damage

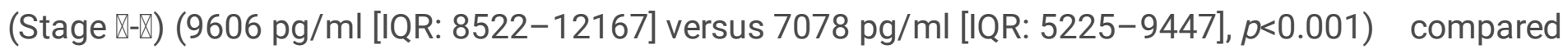
to other patients. Although serum levels of galectin-9 correlated with the titers of ACPA $(r=0.275$, $p=0.002$ ), levels of ACPA titers conferred the different relationship, between serum Gal- 9 and inflammatory mediators or RA disease activity. Although Gal-9 was correlated with ACPA titers $(r=0.508$, $p=0.002)$, there was no correlation between Gal-9 levels and erythrocyte sedimentation rate (ESR), matrix metalloproteinase-3 (MMP-3) or DAS28-ESR in RA patients with high titers of ACPA $(>200 \mathrm{u} / \mathrm{ml})$. Conversely, Gal-9 was correlated with MMP-3 $(r=0.300, p=0.007)$ or DAS28-ESR $(r=0.331, p=0.004)$ not with ACPA titer in RA patients with low titers of ACPA titers (<200U/ml).

Conclusions: Serum levels of glectin-9 were increased in RA patients and associated with RA disease activity in RA patients without high titers of ACPA. The levels of ACPA titers may influence the values of circulating galectin-9 in RA patients with various clinical phenotype. These data suggest that Gal-9 possessed the properties proinflammatory or arthropathic biomarker under the status of ACPA seropositivity,

\section{Introduction}

Rheumatoid arthritis (RA) is an autoimmune disease characterized by synovial inflammation, bone destruction and extra-articular symptoms [1]. Multiple genes, proteins and cells have been identified to contribute to the pathogenesis of RA [2]. A common features of RA is the hyper-activated state of the stromal synovial cells and the immune cells [3]. Dysregulated innate and adaptive pathways are involved in the RA pathogenesis [4]. Galectins are the family members of lectins that expressed on the cell surface or extracellular matrix and bind to $\beta$-galactoside carbohydrates on the cell surface [5]. Through binding to their receptors, galectins play an important role in the pathological processes including inflammation and autoimmunity [6]. Recent studies suggest that Galectins play important roles in the pathogenesis of RA [7]. It was demonstrated that Gal-3 is increased in early RA and associated with anti-CCP seropositivity 
and MRI bone erosion scores in patients with RA [8]. These findings suggest that galectin family play an important role in the disease development of RA, though their interactions with innate or adaptive immunity.

Galectin-9 is expressed by immune cells, endothelial cells and fibroblasts and plays an important role in regulating inflammation and immune reactions [9]. Galectin-9 is a ligand of T cell immunoglobulin and mucin-containing-moleculte-3 (Tim-3) which is expressed on CD $4^{+}$T helper (Th) 1 and Th17 and providing inhibitory signals through its interaction with Tim-3 [10]. Therefore, galectin-9 negatively regulates proinflammatory $T$ cell responses through the interaction with Tim-3 and Gal-9/Tim-3 pathway induces apoptosis of CD $4^{+}$Th1 or Th17 cells [11]. In mouse models, Gal-9 deficiency led to increased number of Th1 and Th17 cells and decreased number of Treg cells in the joint, rendering susceptibility to collagen-induced arthritis (CIA) [12]. Conversely, administration of galectin-9 ameliorated arthritis in CIA and immune-complex-induced murine arthritis model suggesting that Gal-9 prevent the disease progression of RA [13]. Considering that RA is regarded as a Th1-polarized autoimmune disease, dysregulated Gal-9 levels may cause the imbalance in the innate/adaptive immunity, thereby inducing pathological rheumatoid inflammation. We hypothesized that Gal-9 may play a role in the pathogenesis of RA. In this study, we examined the levels of serum Gal-9 in patients with RA and evaluated the results with respect to the clinical parameters.

\section{Methods}

\section{Patients}

This observational single-center study included 116 consecutive RA patients. Patients were enrolled between February 2012 and September 2019, with follow-up ending in September 2019. We retrospectively reviewed the records of these RA patients. All patients were treated in Department of Rheumatology, Fukushima Medical School from June 2009 to March 2019. All the patients met the 2010ACR/EULAR classification criteria for the disease [14]. Probable RA or overlap syndromes were excluded.

The following clinico-demographic data were collected from the Medical Records Unit at Fukushima University Hospital: age, age at onset of RA, gender, Disease Activity Score-28 for Rheumatoid Arthritis with erythrocyte sedimentation rate (ESR) (DAS28-ESR) score [15] and extra-articular manifestations. High-resolution computed tomography (HRCT) imaging of chest were evaluated by blinded radiologists to assess the evidence of RA-related interstitial lung disease (ILD) manifestations. This study was conducted in accordance with the principles of the Declaration of Helsinki. Ethical approval for this study (No. 2019097) was provided by the Ethics Committee of Fukushima Medical University.

\section{Measurement of clinical disease activity}

All patients underwent clinical assessment at baseline, including 28-joint swollen and tender joint counts (28-SJC and 28-TJC, respectively), physician and patient global assessment with visual analogue scales 
$(0-100 \mathrm{~mm})$ and ESR $(\mathrm{mm})$. The composite disease activity indices were subsequently calculated: DAS28-ESR [15]. Result of this score was reported in quantitative value divided into 4 categories: remission with score of $<2.6$, mild activity if score of $\geq 2.6$ to $<3.2$, moderate activity if score of $\geq 3.2$ to $<5.1$ and high activity if score of $\geq 5.1$. Serum MMP-3 levels were measured by latex immunoassay (Panaclear MMP-3 'Latex'; Sekisui Medical Company Limited, Tokyo, Japan). The patients' anti-CCP antibodies were analyzed using commercially available second-generation chemiluminescent enzyme immunoassay kits (STACIA® MEBLux ${ }^{\text {TM }}$ CCP test, Medical and Biological Laboratories, Aichi, Japan) according to the manufacturer's instructions. The results were reported qualitatively where negative or positive for anti-CCP antibody was defined as $<20.0 \mathrm{U} / \mathrm{mL}$ or $\geq 20.0 \mathrm{U} / \mathrm{mL}$, respectively. Radiographs were taken of both hands of each patient. Two rheumatologists, blinded to the patient's identify and functional status, independently graded each hand radiographs and assigned as Steinbrocker radiographic stage [16].

\section{ELISA methods}

Serum concentrations of Galectine-9 were measured using enzyme-linked immnunosorbent assay kit (R\&D Systems, Minneapolis, MN, USA) according to the manufacturer's instruction.

\section{Statistical analysis}

Results were non-normally distributed and are presented throughout the manuscript with median and 2575th centiles [median, IQR] and were compared by the Mann-Whitney $\mathrm{U}$ test. Correlations between continuous variables were analyzed by the Spearman's rank correlation test. All data entry and statistical analyses were performed using SPSS Statistics version 22.0 (IBM, Armonk, NY). In all the analyses, a 2tailed $p<0.05$ was considered statistically significant.

\section{Results}

We recruited 116 patients with RA and 31 gender and age-matched healthy subjects. The demographics and clinical characteristics of the RA patients are presented in Table 1. Among 116 patients, 83 patients (71.6\%) were female. The median age of RA patients was 66 years. The majority of RA patients received DMARDs, mostly methotrexate or MTX in combination with other synthetic DMARDs. Despite the treatments, a median-DAS28-ESR score of 2.8.

We measured serum levels of Gal-9 in RA patients using a specific ELISA assay. As shown in Figure. 1, serum Gal-9 concentrations in patients with RA were significantly higher compared to those in healthy subjects (median $7577 \mathrm{pg} / \mathrm{ml}$; [interquartile range (IQR); 5570-10201] versus $4738 \mathrm{~g} / \mathrm{ml}$ [IQR; 42675630], $p=0.001$ ). Although there was no significant difference in serum levels of Gal-9 between RA patients with and without smoking history (Fig. 2A, $p=0.615$ ), higher levels of serum Gal-9 were observed predominantly in RA patients with RA-ILD (9606 pg/ml [IQR: 8522-12167] versus $7078 \mathrm{pg} / \mathrm{ml}$ [IQR: 5225-9447], $p<0.001$ ) (Fig. 2B). 
We investigated the relationship between serum Gal-9 and each parameters of RA patients. Serum Gal-9 were significantly correlated with ESR $(r=0.344, p<0.001)$, MMP-3 $(r=0.234, p=0.004)$ and ACPA titers $(r=0.275, p=0.002)$. Also serum Gal-9 was significantly correlated with RA disease activity scores, DAS28-ESR $(r=0.269, p=0.005)$.

To further evaluate the ability of serum Gal-9 to differentiate RA phenotype, we analyzed the distribution pattern of serum Gal-9 values in combination with ACPA titer (Fig. 3A). When RA patients were grouped according to the presence of high titers ACPA ( $\geqq 200 \mathrm{U} / \mathrm{ml}$ ), some correlations between circulating Gal-9 and clinical features were identified. In the two dimensional heat-nap consisting serum Gal-9 and ACPA titer, we identified two groups (Fig. 3A). Group1 RA patients exhibited high ACPA titers (ACPA $\geqq 200 \mathrm{U} / \mathrm{ml}$ ), and Group 2 RA patients exhibited moderate to low ACPA titers (ACPA $<200 \mathrm{U} / \mathrm{ml}$ ). There was a significant modest correlation between Gal-9 and ACPA titers in Group1 RA patients $(r=0.508, p=0.002)$. Whereas there was no correlation between Gal-9 and ACPA titer in Group 2 RA patients $(r=0.211, p=$ 0.060) suggesting that Gal-9 was not modulated by the status of ACPA titers (Fig. 3B). Next we evaluated the correlations between Gal-9 and clinical parameters in subdivided Group 1 or Group 2 RA patients. A significant correlations between circulating Gal-9 and inflammatory markers, ESR $(r=0.451, p<0.001)$ or DAS28-ESR $(r=0.331, p=0.004)$ were identified in RA patients with low titers of ACPA $(<200 \mathrm{U} / \mathrm{ml})$, whereas there was no correlation between circulating Gal-9 and these parameters $(r=0.203, p=0.249$ and $r=0.160, p=0.365$ ) in RA patients with high titers of ACPA ( $\geqq 200 \mathrm{U} / \mathrm{ml}$ ) (Fig. 4A, 4B). Similarly, in RA patients with high titers of ACPA ( $\geqq 200 \mathrm{U} / \mathrm{ml}$ ), there was no correlation between serum Gal-9 and MMP-3 $(r=0.111, p=0.519)$, whereas serum Gal-9 levels were significantly correlated with serum MMP-3 $(r=$ 0.300. $p=0.007)$ in RA patients with low titers of ACPA $(<200 \mathrm{U} / \mathrm{ml})$ (Fig. 4C). These findings suggest that serum Gal-9 were upregulated in link to autoimmune response in RA patients with high titer ACPA. Whereas serum Gal-9 was upregulated in response to inflammatory mediators in RA patients without low titers of ACPA.

From a clinical point of view, the circulating Gal-9 were compared according to the presence or absence of clinical remission in these sub-grouped RA patients (Fig. 5). In RA patients low titers of ACPA (< $200 \mathrm{U} / \mathrm{ml}$ ), circulating Gal-9 were significantly higher in patients without clinical remission compared to those with clinical remission (8252 pg/ml [IQR: 5870-10996] versus $7103 \mathrm{pg} / \mathrm{ml}$ [IQR: 5328-8357], $\mathrm{p}=$ $0.013)$. Whereas, there was no significant difference in circulating Gal-9 between patients with and without clinical remission (10647 pg/ml [IQR: 6960-13367] versus $8635 \mathrm{pg} / \mathrm{ml}$ [IQR: 6372-10092], $\mathrm{p}=$ $0.703)$ in RA patients with high titers of ACPA ( $\geqq 200 \mathrm{U} / \mathrm{ml}$ ).

Finally, we subdivided RA patients according to the progressing of joint damage (Stage) and we evaluated the relationship between serum Gal-9 and progressive joint damage. As shown in Fig. 6A, RA patients with advanced articular lesions (Stage $\otimes-\varangle$ ) had significantly higher levels of circulating Gal-9 compared to those without advanced articular lesions (Stage I) (8790 pg/ml [IQR: 5631-10953] versus $7103 \mathrm{pg} / \mathrm{ml}$ [IQR: 5882-8810], $\mathrm{p}=0.023$ ). There was a significant difference in circulating Gal-9 between those with and without advanced articular lesions (Stage $\mathbb{X}-\mathrm{X}$ ) in RA patients low titers of ACPA (7367 pg/ml [IQR: 5931-10109] versus 7009 pg/ml [IQR: 4602-9450] $\mathrm{p}=0.004$ ) Whereas three was no 
significant difference in circulating Gal-9 between those with and without advanced articular lesions (Stage $\mathbb{X}-\mathbb{Z}$ ) in RA patients with high titers of ACPA (10146 pg/ml [IQR: 8771-12257] versus $5882 \mathrm{pg} / \mathrm{ml}$ [IQR: 5053-8138], $p=0.182$ ) (Fig. 6B).

\section{Discussion}

In this study we evaluated circulating levels of Gal-9 in established RA patients with various disease activity. We demonstrated that Gal-9 is significantly elevated in RA patients and correlated with the titers of ACPA as as well as rheumatoid inflammatory markers such as ESR or MMP-3. Whereas we could not find positive correlations between Gal-9 concentrations and MMP-3 or DAS-28-ESR in sub-grouped RA patients with high titers of ACPA $(>200 \mathrm{u} / \mathrm{ml})$. These results suggest that galectin-9 could be biomarkers estimating RA disease activity under the particular ACPA status.

There is the evidences that the pathogenesis of RA differs between ACPA-positive and ACPA-negative RA patients [17]. This antibody could be used as a prognostic factor, since high titers for ACPA is associated with worse radiographic progression [18]. It was also postulated that an association between seropositivity of ACPA and response to abatacept (ABT) could be caused the blocking the interaction between T cells and APC via costimulatory molecules in which the effects of ABA on T cell costimulation is more marked in ACPA-positive patients [19]. These data suggest that RA patients with high-titer of ACPA represent a unique RA population.

We found a positive correlation between circulating Gal-9 and the inflammatory mediators in RA patients suggesting that the dysregulated circulating Gal-9 is involved in RA pathophysiology as a modulator for autoimmunity as well as rheumatoid inflammation. Furthermore, differential patterns of ACPA seropositivity and inflammatory mediators have implications in the increased circulating Gal-9 in RA patients. Gal-9 has been suggested to play a role in RA pathogenesis, but the underlying mechanisms have not been elucidated. Previous studies suggest that the measurement of disease activity alone is not sufficient to identify fast progressing RA and high titers of ACPA could be a risk factor for RA progression $[20,21]$. The correlation between Gal-9 and ACPA observed in our study may suggest that high titers of ACPA may be linked to the rheumatoid inflammatory process through the Gal-9-mediated bone effects indirectly. Interestingly, we found increased serum levels of Gal-9 in RA patients with progressive joint damage. Galectin family is involved in the rheumatoid osteoclastogenesis and inflammatory bone destruction [22]. Expression of Tim-3 was demonstrated in osteoclasts and Gal-9 markedly inhibited osteoclastogenesis via Tim-3/Gal-9 system [23]. Therefore, it is possible that the Tim-3/Gal-9 system may regulate the rheumatoid inflammatory bone destruction. Taken together the elevated circulating levels of Gal-9 seen in RA patients may reflect the augmented status of osteoclastogenesis of RA joints. Whereas it was reported that unlike to exogenous Gal-9, endogenous Gal-9 is protective against apoptosis and enhances synovial fibroblast viability suggesting the pathogenic and pro-inflammatory role in RA [24]. Considering the complex biological role of Gal-9, further studies are needed to determine the mechanism for the increased Gal-9 expression and its function upon the disease activity and joint damage of RA patients. 
More recently, Wiersma et al reported that serum Gal-9 levels were elevated and correlated with disease activity (DAS-28) or smoking status in RA patient [25]. Our data are almost in line with these data. Serum Gal-9 levels were elevated in RA patients with RA-related interstitial lung disease (RA-ILD), whereas, we could not find any associations between circulating Gal-9 and smoking status in our RA patients. Differences of ethnics and demographic data of the subjected RA patients may contribute to the differential result between our data and the reported data [25]. Further, large-scale investigations consisting of ethnic-groups are needed to decide the mechanisms by which circulating Gal-9 is elevated in RA patients.

Variation of disease course and treatment response in RA patients originate from the heterogeneity of this syndrome [26]. ACPA-positive and ACPA-negative disease have been shown to be associated with different genetic or environmental factors suggesting that different pathological mechanisms are implicated in these two separate disease subsets [27]. It is presumed that stratifying patients with RA on the basis of ACPA and Gal-9 status enables to identify more homogenous RA phenotype with respect to disease activity or joint structure damages.

There are several potential limitations of this study that should be considered. First, the patient population was relatively small and a larger study is essential to confirm our results. Second, all patients with RA and healthy individuals in this study were Japanese, additional studies in other ethnic groups are needed to verify these findings. Third, the mechanism by which Gal-9 contributes to the pathogenesis of RA was not clarified. Finally, it will be important to examine the longitudinal changes of serum galectin-9 levels in patients with RA and to assess their extraarticular involvements in the future studies. Nevertheless, our findings suggest that Gal-9 may be involved in the pathophysiology of RA reflecting disease activity or immune phenotypes of RA.

\section{Conclusions}

Serum Gal-9 shows as an additional marker for evaluating disease activity in patients with RA. Prospective investigation of the combination of Gal-9 and ACPA may facilitate development of diagnostic tools to assess disease activity and disease phenotype in patients with RA.

\section{Declarations}

\section{Ethical Approval and Consent to participate}

Ethical approval for this study (No. 2019097) was provided by the Ethics Committee of Fukushima Medical University and written informed consent was obtained from each individual.

\section{Consent for publication}

Not applicable 
Availability of supporting data

Not applicable

\section{Competing interests}

KM has received research grants from Chugai, Pfizer, and AbbVie. Rest of the authors declares that they have no competing interests

\section{Funding}

The study was supported by the Japan Grant-in-Aid for Scientific Research(C 17K09981).

\section{Authors' contributions}

YF, TA, NM, JT, SS, HM, MF, ES, HK, HW were involved in acquisition of clinical data and drafted manuscript. YF performed the statistical analysis. KM participated in the design of the study and drafted manuscript. All authors read and approved the final manuscript.

\section{Acknowledgements}

We are grateful to Ms Kanno Sayaka for her technical assistance in this study.

\section{Abbreviations}

ACPA=Anti-citrullinated peptide antibodies

ESR=erythrocyte sedimentation rate

Gal-9=Galectin-9

$\mathrm{RA}=$ rheumatoid arthritis (RA)

MMP-3=matrix metalloproteinase-3

\section{References}

1. Smolen JS, Aletaha D, Mclnnes IB. Rheumatoid arthritis. 2016;388(10055):2023-2038.

2. Jutley GRaza K, Buckley CD. New pathogenic insights into rheumatoid arthritis. Curr Opin Rheumatol. 2015;27(3):249-55.

3. Bartok B, Firestein GS. Fibroblast-like synoviocytes: key effector cells in rheumatoid arthritis. Immunol Rev. 2010;233(1):233-55.

4. Catrina Al, Joshua V, Klareskog L, Malmström V. Mechanisms involved in triggering rheumatoid arthritis. Immunol Rev. 2016;269(1):162-74. 
5. Thiemann S, Baum LG. Galectins and Immune Responses-Just How Do They Do Those Things They Do? Annu Rev Immunol. 2016;34:243-64.

6. Rabinovich GA, Rubinstein N, Toscano MA. Role of galectins in inflammatory and immunomodulatory processes. Biochim Biophys Acta. 2002;1572(2-3):274-84.

7. Ilarregui JM, Bianco GA, Toscano MA, Rabinovich GA. The coming of age of galectins as immunomodulatory agents: impact of these carbohydrate binding proteins in T cell physiology and chronic inflammatory disorders. Ann Rheum Dis. 2005;64 Suppl 4:iv96-103.

8. Issa SF, Christensen AF, Lindegaard HM, et al. Galectin-3 is Persistently Increased in Early Rheumatoid Arthritis (RA) and Associates with Anti-CCP Seropositivity and MRI Bone Lesions, While Early Fibrosis Markers Correlate with Disease Activity. Scand J Immunol. 2017;86(6):471-478.

9. Gieseke F, Kruchen A, Tzaribachev N, Bentzien F, Dominici M, Müller I. Proinflammatory stimuli induce galectin-9 in human mesenchymal stromal cells to suppress T-cell proliferation. Eur $\mathrm{J}$ Immunol. 2013;43(10):2741-9.

10. Zhu C, Anderson AC, Kuchroo VK. TIM-3 and its regulatory role in immune responses. Curr Top Microbiol Immunol. 2011;350:1-15.

11. Zhu C, Anderson AC, Schubart A, et al. The Tim-3 ligand galectin-9 negatively regulates $T$ helper type 1 immunity. Nat Immunol. 2005;6(12):1245-52.

12. Arikawa $T$, Watanabe $K$, Seki M, et al. Galectin- 9 ameliorates immune complex-induced arthritis by regulating Fc gamma R expression on macrophages. Clin Immunol. 2009;133(3):382-92.

13. Seki M, Sakata KM, Oomizu S, et al. Beneficial effect of galectin 9 on rheumatoid arthritis by induction of apoptosis of synovial fibroblasts. Arthritis Rheum. 2007;56(12):3968-76.

14. van der Linden MP, Knevel R, Huizinga TW, van der Helm-van Mil AH. Classification of rheumatoid arthritis: comparison of the 1987 American College of Rheumatology criteria and the 2010 American College of Rheumatology/European League Against Rheumatism criteria. Arthritis Rheum. 2011;63(1):37-42.

15. Fransen J, van Riel PL. The Disease Activity Score and the EULAR response criteria. Rheum Dis Clin North Am. 2009 Nov;35(4):745-57, vii-viii.

16. Steinbrocker $\mathrm{O}$, Traeger $\mathrm{CH}$, Batterman RC. Therapeutic criteria in rheumatoid arthritis. J Am Med Assoc. 1949;140:659-62.

17. Willemze A, Trouw LA, Toes RE, Huizinga TW. The influence of ACPA status and characteristics on the course of RA. Nat Rev Rheumatol. 2012;8(3):144-52.

18. Degboé $Y$, Constantin A, Nigon D, et al. Predictive value of autoantibodies from anti-CCP2, anti-MCV and anti-human citrullinated fibrinogen tests, in early rheumatoid arthritis patients with rapid radiographic progression at 1 year: results from the ESPOIR cohort. RMD Open. 2015;1(1):e000180.

19. Sokolove J, Schiff M, Fleischmann R, et al. Impact of baseline anti-cyclic citrullinated peptide-2 antibody concentration on efficacy outcomes following treatment with subcutaneous abatacept or adalimumab: 2-year results from the AMPLE trial. Ann Rheum Dis. 2016;75(4):709-14. 
20. Sewerin P, Vordenbaeumen S, Hoyer A, et al. Silent progression in patients with rheumatoid arthritis: is DAS28 remission an insufficient goal in RA? Results from the German Remission-plus cohort. BMC Musculoskelet Disord. 2017;18:163.

21. Steffen U, Schett G, Bozec A. How Autoantibodies Regulate Osteoclast Induced Bone Loss in Rheumatoid Arthritis. Front Immunol. 2019;10:1483.

22. Moriyama K, Kukita A, Li YJ, et al. Regulation of osteoclastogenesis through Tim-3: possible involvement of the Tim-3/galectin-9 system in the modulation of inflammatory bone destruction. Lab Invest. 2014;94(11):1200-11.

23. Li YJ, Kukita A, Teramachi J, et al. A possible suppressive role of galectin-3 in upregulated osteoclastogenesis accompanying adjuvant-induced arthritis in rats. Lab Invest. 2009;89(1):26-37.

24. Pearson MJ, Bik MA, Ospelt C, et al. Endogenous Galectin-9 Suppresses Apoptosis in Human Rheumatoid Arthritis Synovial Fibroblasts. Sci Rep. 2018;8(1):12887.

25. Wiersma VR, Clarke A, Pouwels SD, et al. Galectin-9 Is a Possible Promoter of Immunopathology in Rheumatoid Arthritis by Activation of Peptidyl Arginine Deiminase 4 (PAD-4) in Granulocytes. Int J Mol Sci. 2019;20(16).

26. England BR, Thiele GM, Mikuls TR. Anticitrullinated protein antibodies: origin and role in the pathogenesis of rheumatoid arthritis. Curr Opin Rheumatol. 2017;29(1):57-64.

27. Catrina Al, Joshua V, Klareskog L, Malmström V. Mechanisms involved in triggering rheumatoid arthritis. Immunol Rev. 2016;269(1):162-74.

\section{Table}

Baseline demographics, clinical characteristics, and profiles ( $\mathrm{n}=116)$

Characteristics Value

Age, years (mean \pm SD)

Female, n (\%)

Smoker, n (\%)

RA-ILD, n (\%)

Duration of RA, year, (mean \pm SD)

ESR, $\mathrm{mm} / \mathrm{h}$, (mean \pm SD)

CRP, mg/dL, (mean \pm SD)

MMP-3, ng/mL, (mean \pm SD)

$\mathrm{RF}, \mathrm{IU} / \mathrm{mL}$, (mean $\pm \mathrm{SD})$

Anti CCP-Ab, U/mL, (mean \pm SD)

Corticosteroid, n (\%)

Methotrexate, n (\%)

Biologics, n (\%)

DAS28-ESR, (mean \pm SD)

Steinbrocker stage

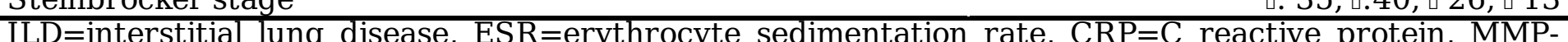

$3=$ matrix metalloproteinase-3, RF=rheumatoid factor, CDAI=Clinical Disease Activity index, SDAI=simplified disease activity index, DAS28=Disease Activity Score, SD=standard deviation

\section{Figures}




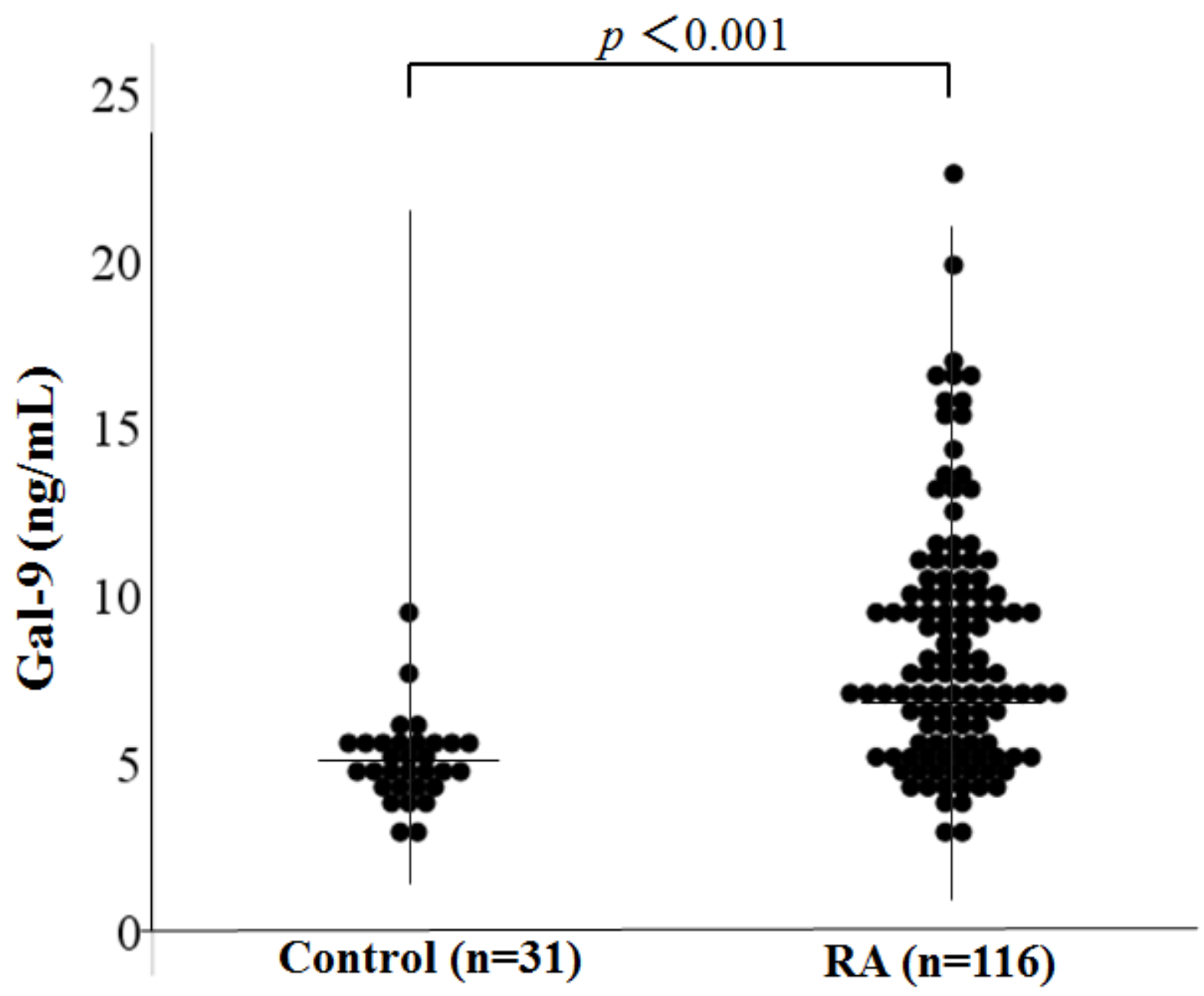

Figure 1

Serum levels of Galectin-9 in RA patients and controls Serum levels of Galectin-9 in RA patients $(n=116)$ were significantly higher compared to those in healthy subjects $(n=31)$. 
A

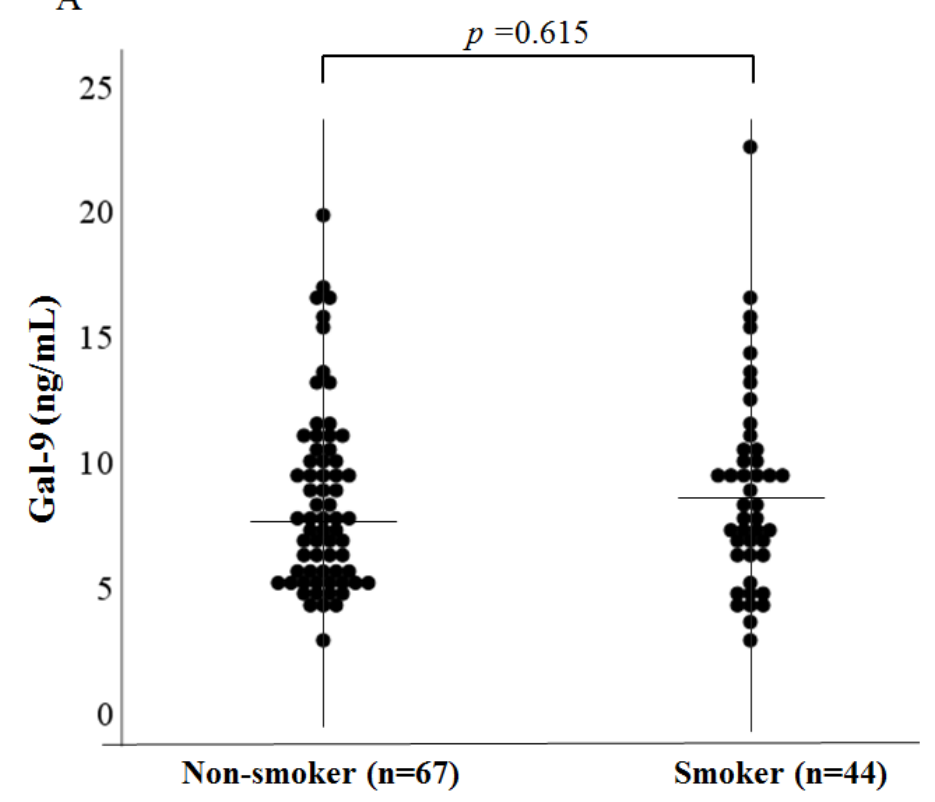

B

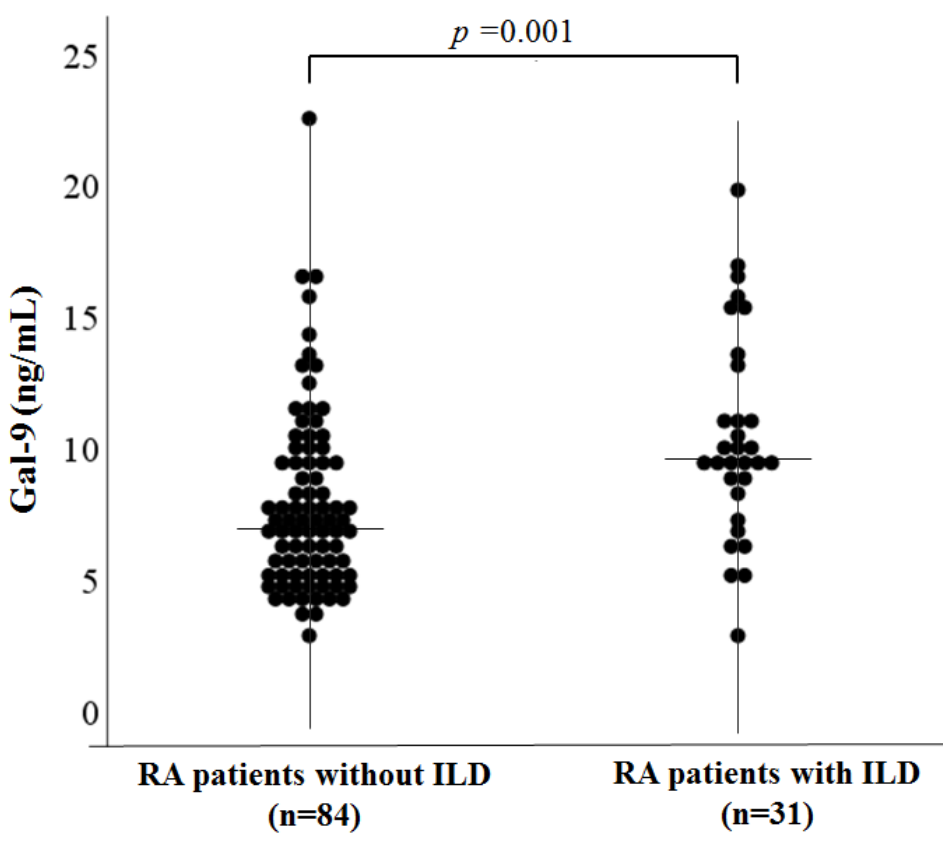

Figure 2

Figure 2A Serum levels of Galectin-9 in RA patients with or without RA-related interstitial lung disease (ILD) We compared serum levels of Galectin-9 between RA patients with or without RA-related ILD. Serum levels of Galectin-9 were significantly higher in patients with RA-related ILD compared to those without RA-related ILD. Figure 2B Serum levels of Galectin-9 in RA patients with or without smoking history We compared serum levels of Galectin-9 between RA patients with or without smoking history. There was no significant difference in serum levels of Galectin-9 between RA patients with and without smoking history. 

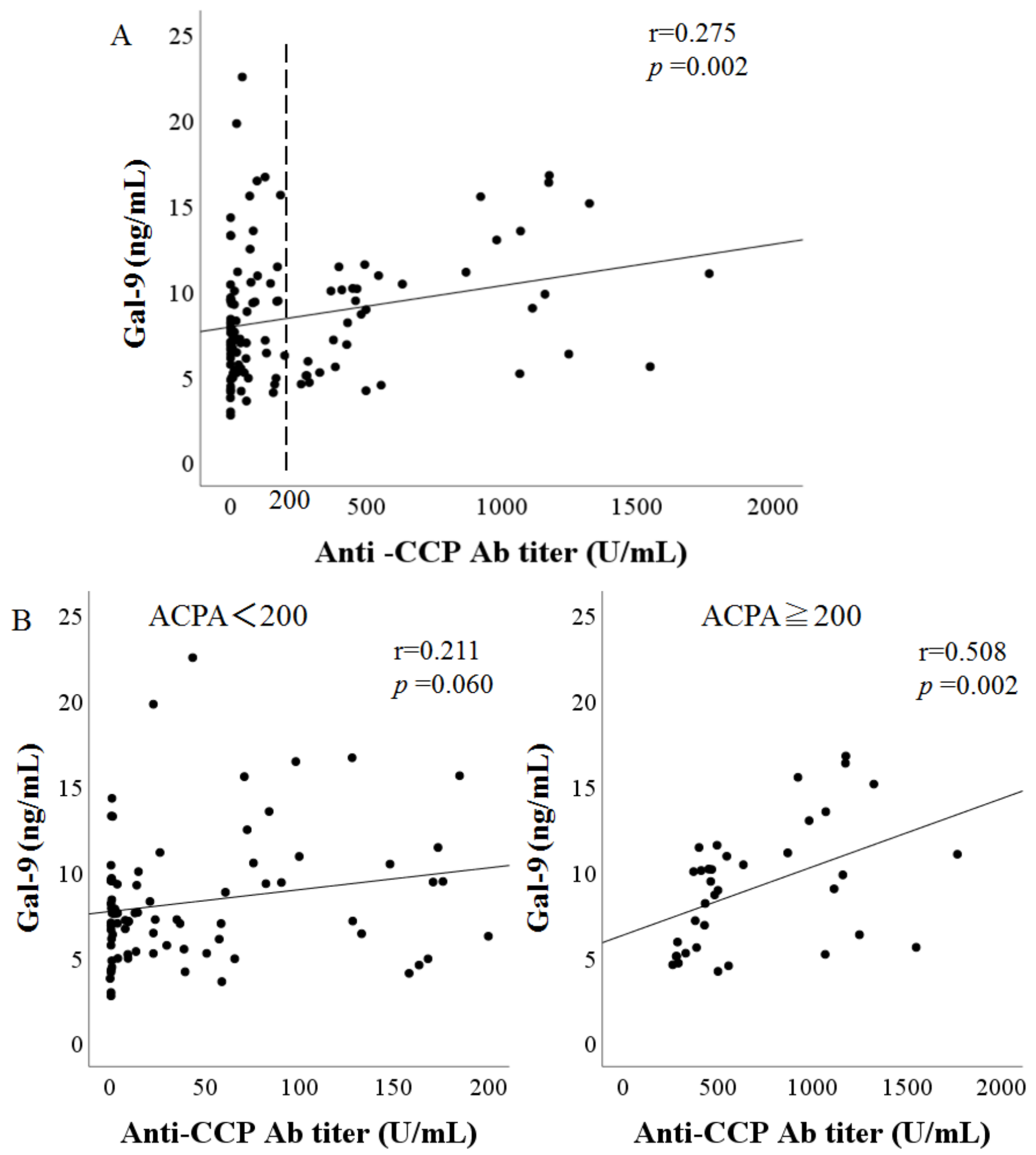

Figure 3

Relationship between anti-cirtrullinated peptide antibody (ACPA) titers and serum levels of Galectin-9 in patients with rheumatoid arthritis (RA) A: Levels of ACPA titers were measured and correlation analysis with serum levels of Galectin-9 was performed. B: Correlation analysis of serum levels of Galectin-9 and

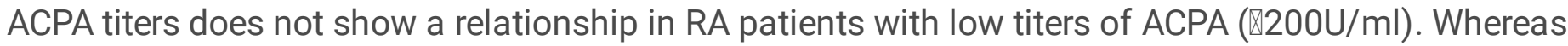


there was a significant positive correlation between serum levels of Gal-9 and ACPA titers in RA patients with high titers of ACPA ( $\geqq 200 \mathrm{U} / \mathrm{ml})$.
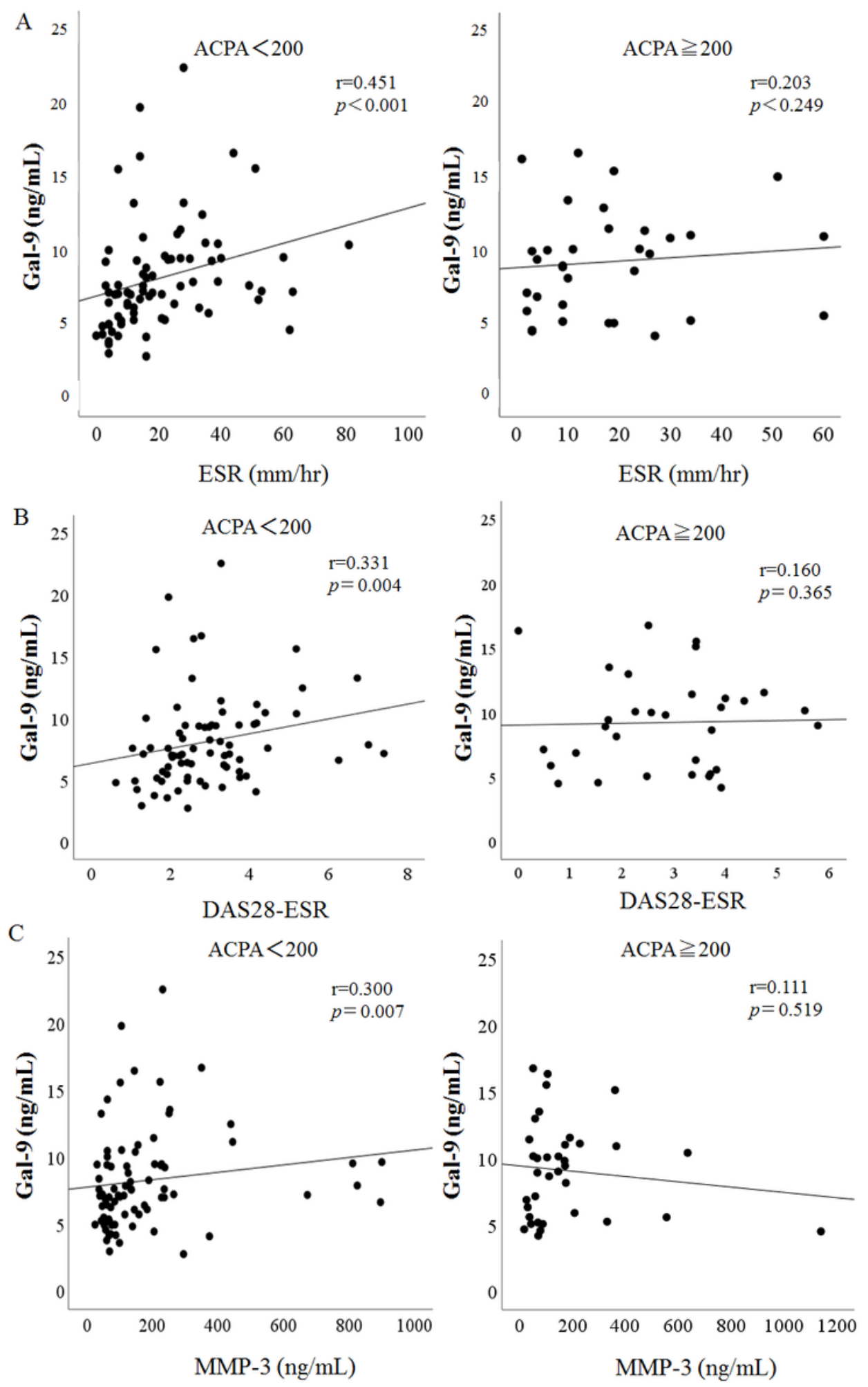

Figure 4

Correlation between serum levels of Galectin-9 and clinical parameters (A: ESR, B: DAS28-ESR, C:MMP-3) in the sub-grouped RA patients according to the titers of ACPA A: Correlation analysis of serum levels of Galectin-9 and ESR does not show a relationship in Group 1 RA patients with high titers of ACPA 
( $\geqq 200 \mathrm{U} / \mathrm{ml}$ ). Whereas there was a significant positive correlation between serum levels of Galectin-9 and

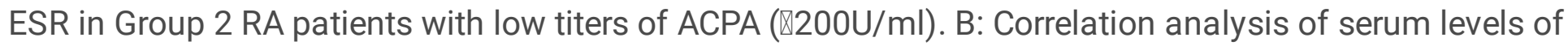
Galectin-9 and DAS28-ESR does not show a relationship in Group 1 RA patients with high titers of ACPA $(\geqq 200 \mathrm{U} / \mathrm{ml}$ ). Whereas there was a significant positive correlation between serum levels of Galectin-9 and

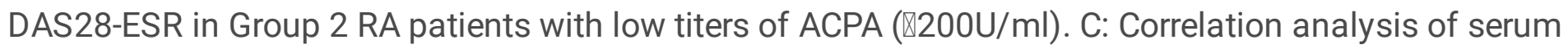
levels of Galectin-9 and MMP-3 does not show a relationship in Group 1 RA patients with high titers of ACPA ( $\geqq 200 \mathrm{U} / \mathrm{ml}$ ). Whereas there was a significant positive correlation between serum levels of Galectin9 and MMP-3 in Group 2 RA patients with low titers of ACPA ( $(200 \mathrm{U} / \mathrm{ml})$.
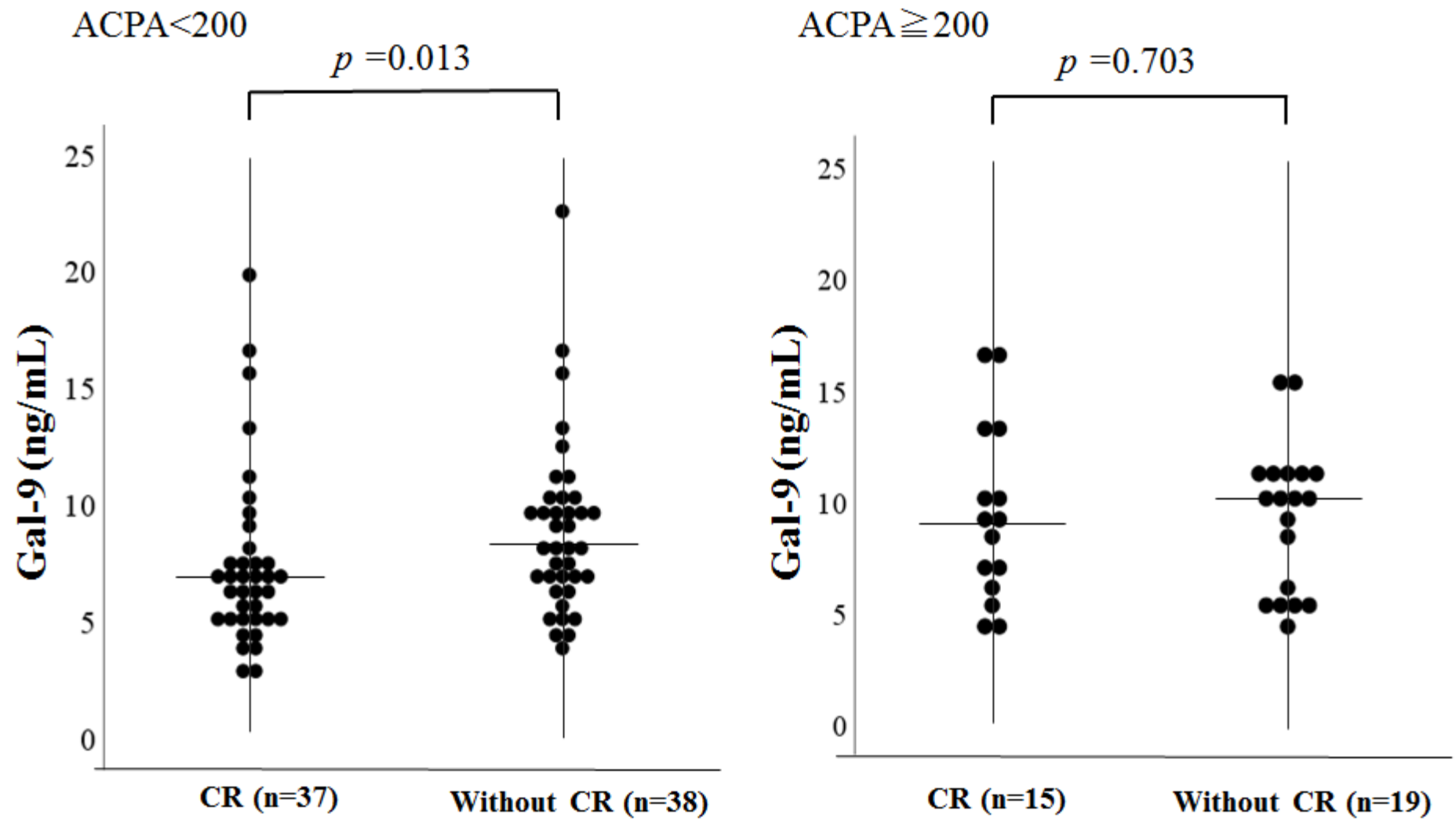

Figure 5

Serum levels of Galectin-9 in RA patients with or without DAS28-ESR clinical remission (CR). We compared serum levels of Galectin-9 between RA patients with or without clinical remission (CR). Serum levels of Galectin-9 were significantly lower in patients with CR compared to those without CR in RA

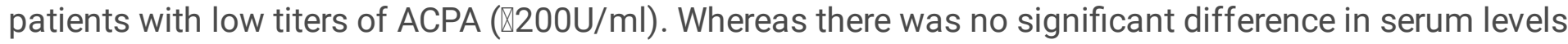
of Galectin-9 between patients with and without CR in RA patients with high titers of ACPA ( $\geqq 200 \mathrm{U} / \mathrm{ml})$. 
A

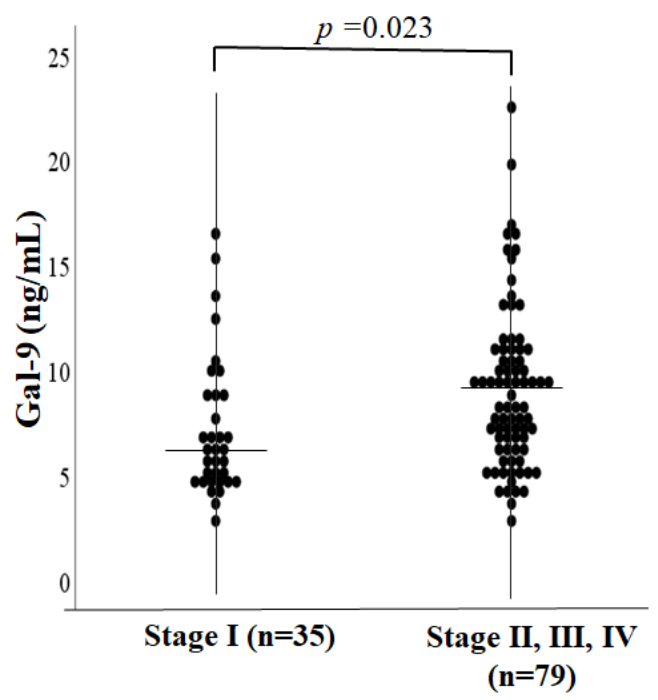

$\mathrm{B}$

ACPA $<200$

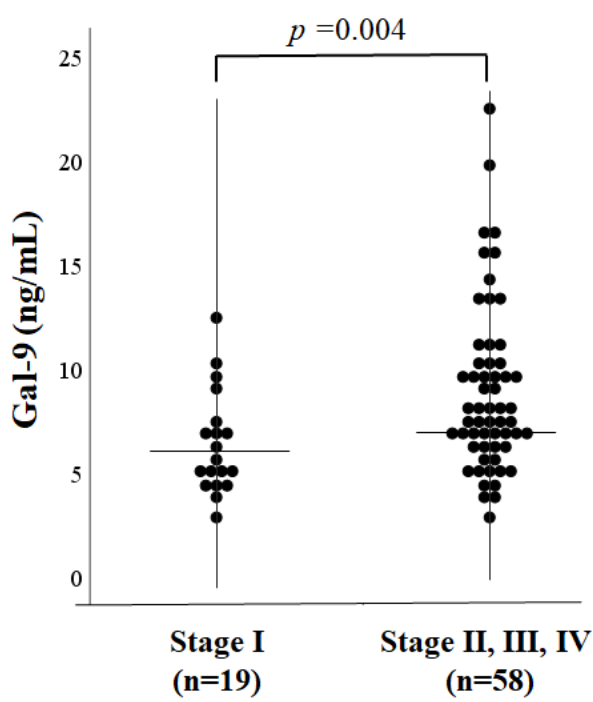

$\mathrm{ACPA} \geqq 200$

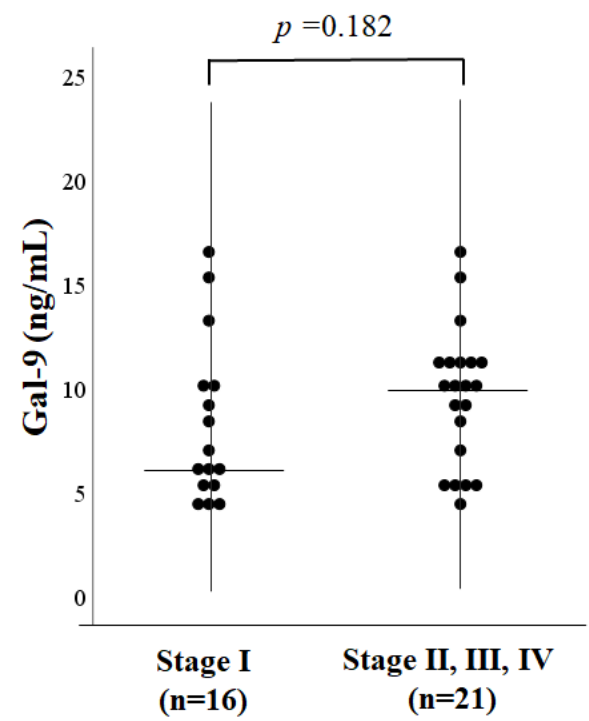

Figure 6

Serum levels of Galectin-9 in RA patients with or without advanced joint damage. We compared serum

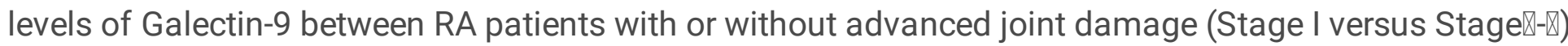
A: Serum levels of Galectin-9 were significantly higher in RA patients with advanced joint damage (Stage $\mathbb{Q}-\mathbb{Z}$ ) compared to those without advanced joint damage (Stage I). B: We compared serum levels of

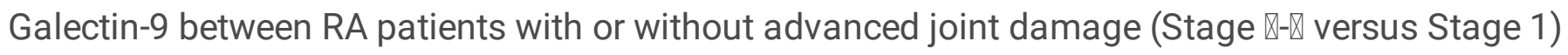
according to the ACPA titers. Serum levels of Galectin-9 were significantly higher in patients with

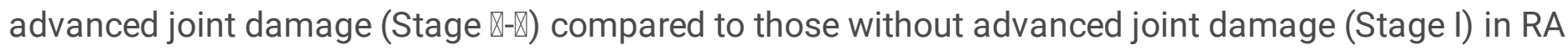

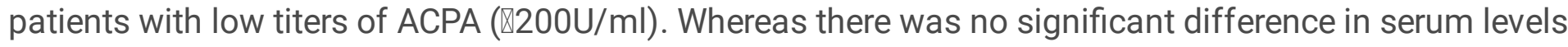
of Galectin-9 between patients with and without advanced joint damage in RA patients with high titers of ACPA ( $\geqq 200 \mathrm{U} / \mathrm{ml})$. 